\title{
A real option methodology to determine the optimal intervention windows for railway infrastructure
}

\author{
N. Lethanh \& B. T. Adey \\ Institute of Construction and Infrastructure Management, \\ ETH Zürich, Switzerland
}

\begin{abstract}
In this paper, a real option methodology is presented for the determination of the optimal expected time in the future for a railway infrastructure manager to decide what types of interventions, if any, are to be executed. This time is herein referred to as the optimal time to decide to execute an intervention to emphasize that when the time is fixed it is not yet known if interventions will actually be executed. Such a methodology is particularly useful in the management of railway infrastructure that belongs to a multinational freight corridor where multiple railway management organizations are involved. Every time one executes an intervention it can affect the flow of trains on the part of the infrastructure controlled by others. In the methodology an adaptation of the model used to value options in financial engineering using the Black and Scholes differential equation is used. The model enables the valuation of the ability to wait to decide to determine whether or not an intervention should be executed. The methodology is demonstrated by determining the optimal time to decide to execute interventions on a fictive rail corridor for a railway management organization. Use of the methodology is expected to improve the coordination of the execution of interventions on multiple parts of the corridor, and give a period of time in which it is relatively certain that no interventions will be executed.
\end{abstract}

Keywords: maintenance, rail infrastructure, real options. 


\section{Introduction}

Management of railway infrastructure that belongs to a national track train system or a multinational freight corridor (e.g. the trans-Europe transportation network comprises six freight corridors [1]) requires railway managers to execute interventions to ensure that an adequate level of service is provided. As multiple railway management organizations are involved, and every time one executes an intervention it can affect the flow of trains on the part of the corridor controlled by others, it would be desirable for all involved organizations to agree on a time to execute interventions even if it is not known exactly which interventions are to be executed when this time is fixed. This would improve the coordination of the execution of interventions on multiple parts of the corridor, and give a period of time in which it is relatively certain that no interventions will be executed.

As the decision to execute interventions is based on the values of numerous variables with which there is uncertainty, e.g. increases in the number of trains, the deterioration due to the wearing of tracks and the changes in track geometry due to flooding, it is in the best interest of each railway management organization to determine for itself the optimal time to execute interventions even if it is unclear as to the interventions, if any, that will be executed. This optimal time is herein referred to as the optimal time to decide to execute interventions (OTD) to emphasize that when the time is fixed it is not yet known if interventions will actually be executed. It may also be seen as the optimal window in which interventions could be, but must not be, executed.

Recent work has indicated that this is possible using a methodology built on real options (RO), such as proposed by [2] in other civil engineering applications. A methodology built on RO makes it possible to directly take into consideration the probability of obtaining new information in the future and the fact that interventions will only be executed if certain conditions are met.

Most of the work focused on investigating the use of RO methodologies in the field of civil engineering decision making has been focused on decision making related to the construction of new infrastructure, such as the construction of a new airport [3], of a high-speed passenger train system [4], of an electricity distribution network [5], and of a new addition to an existing highway network or in the development of construction projects in general [6]. Almost no work has been focused on decision making related to the maintenance of existing infrastructure, and none on decision making related to the maintenance of railway infrastructure. The former includes the example given by [2] of how a RO methodology can be used in decision making related to the maintenance of offshore structures. Although no work has been done on the latter, however, many researchers are investigating the determination of optimal intervention strategies for rail infrastructure.

In the methodology proposed in this work for the determination of the OTD, an adaptation of the model used to value European call options in financial engineering using the Black and Scholes differential equation is used. The model enables, as do all RO models, the valuation of the ability to wait to decide to 
determine whether or not an intervention should be executed. The methodology is demonstrated by determining the optimal time to decide to execute interventions on a fictive rail corridor for a railway management organization.

The remainder of this paper is structured as follows. The RO model used in the methodology to determine the OTD and the assumptions made in establishing the model parameters are explained in section 2. The RO methodology is shown by conducting an example to determine the OTD for a fictive rail link for a railway management organization in section 3 . Conclusions and discussion are given in section 4.

\section{The model}

The model is developed assuming that a railway management organization is interested in determining the OTD during a finite time period $T$, for an existing railway link. This decision strategy (DS) comprises only one point in time, $z$, in which it can be decided whether an intervention is to be executed or not, and the intervention is executed. The organization is interested in determining the DS that will maximize the total expected net benefits, i.e. optimal $z$ on $0 \leq z \leq T$.

In this model, there exists two possibilities at $z$ : (1) the global condition state (GCS) of the link does not trigger the execution of an intervention, or (2) the GCS of the link triggers the execution of an intervention. If possibility (1) occurs, the probability of the link entering the GCS where an intervention is required increases. If possibility (2) occurs, then the link is restored to an as-new GCS and the deterioration starts over, but not necessarily in the same way. In both cases, once the OTD has passed, it is assumed that limits on train movements, e.g. speed restrictions, are imposed if the GCS, where an intervention is required, is reached.

The time period $(0, T)$ is divided into three parts. The first is from now to the start of the OTD $(0, \mathrm{z})$, the second is during the OTD, and the third is from the end of the OTD to the end of the investigated time period $(z, T)$. It is assumed here for mathematical convenience that the OTD is instantaneous.

The impacts due to routine maintenance and operation before and after $z$ are represented as $R(0, z)$ and $R(z, T)$, and $B(0, z)$ and $B^{d}(z, T)$, respectively. The superscript $d$ refers to intervention type $(d \in D)$, where to do nothing is also seen as an intervention type and $D$ is a set of intervention types (The intervention considered here is the intervention on the link and includes the interventions to be executed on all objects of the link.) The negative impacts incurred during the execution of an intervention, for example due to interrupted train schedules, are represented as $C_{z}^{d}$.

The OTD is then given as:

$$
\begin{aligned}
S(0: T)=\int_{0}^{z} S(0, t) e^{-\rho t} d t+e^{-\rho \cdot z} \int_{z}^{T} S^{d^{*}}(z, T) e^{-\rho t} d t \\
+\operatorname{Max}\left\{e^{-\rho z} \int_{z}^{T}\left[S^{d}(z, T)-S^{d^{*}}(z, T)\right], 0\right\}
\end{aligned}
$$


where, $S(t)=B(t)-R(t)-C(t)$ : is the net benefit. Here, without loss of generality, the superscript and subscript are ignored. The benefit of each DS is formulated as the subtraction of total revenue $B(t)$, routine maintenance $R(t)$, and cost of intervention $C(t) . d^{*}$ is used to denote the execution of a reference intervention, e.g. do nothing, at $z ; \rho$ is discount factor; $B(t)$ includes $B(0, z]$, $B^{d^{*}}(z, T]$, and $B^{d}(z, T]$ and can be expressed as:

$$
B(t)=\sum_{i=1}^{I}\left[N_{i}(t) \cdot h_{i}(t)-O_{i}(t)\right]
$$

where, $N_{i}(t)(i=1, \cdots, I)$ is index representing the quantity of transported goods; $i$ is the index used to indicate the type of goods; $h_{i}(t)$ is unit price of index $i$, per ton of transport goods of type $\mathrm{I} ; O_{i}(t)$ is the operational cost on $i$.

It is implied in Equation (1) that if the execution of intervention $d$ is more beneficial than the execution of intervention $d^{*}$, the intervention will be executed. Otherwise, the intervention $d^{*}$ will be executed.

The values of each of these parameters could be modeled probabilistically, e.g. the prices of electricity and oil. In many cases, they can be modeled as a geometric Brownian motion as:

$$
d h_{i}(t)=\mu_{i, h} \cdot h_{i}(t) \cdot d t+\sigma_{i, h} \cdot h_{i}(t) \cdot d \omega_{i, t}
$$

where, $\mu_{i, h}$ is the drift parameter of the geometric Brownian motion; $\sigma_{i, h}$ represents the standard deviation of the change in the price of $i ; \omega_{i, t}$ is a parameter used to model uncertain variables using the Wiener process. It has zero mean and standard deviation of $\sigma_{i, h} \sqrt{d t}$.

The expected value of uncertain variables given the value at time $t$, e.g. the future price of oil $h_{i}(t+u)$ given the price at time $t h_{i}(t)$ can be described as:

$$
E\left[h_{i}(t+u)\right]=h_{i}(t) \cdot e^{\mu_{i} \cdot u}
$$

where, $u$ is the length of time between $t$ to $t+u$; whenever the link is in a GCS where an adequate level of service is not provided, negative impact is incurred, $W_{i}$, and the value of $B(t)$ is given by:

$$
B(t)=\sum_{i=1}^{I}\left\{\tilde{F}(t)\left[N_{i}(t) \cdot h_{i}(t)-O_{i}(t)\right]-(1-\tilde{F}(t)) \cdot W_{i}\right\}
$$

where, $\tilde{F}(t)$ is the probability of the railway link being in a GCS that does not trigger the execution of an intervention (this is sometimes referred to as survival probability), and $W$ are the negative impacts due to entering a GCS before an 
intervention is executed, e.g. due to the automatic imposition of speed restrictions. Values of $W$ can be determined by using historical data [7].

This type of evaluation is similar to the so called "European call option" in financial engineering, where at a predetermined time $z$, the holder of an option is allowed to make a decision on whether the option will be exercised, but not the obligation to do so.

With such a model it is possible to estimate the probability of a GCS occurring in which an intervention is to be executed using a Weibull function [8]. One of the main advantages of using the Weibull function is that it is not without memory [9]. Using the Weibull function, the functional form of the probability of not entering the triggering GCS $\tilde{F}(t)$ in Equation (5) is:

$$
\tilde{F}(\tau)=\exp \left(-\alpha \tau^{m}\right)
$$

where, $\alpha$ is the so-called arrival density (or failure rate), and $m$ is the acceleration or shape parameter.

Values of parameters $\alpha$ and $m$ can be estimated using regression analysis with available data.

The benefit $S^{d}(t)$ of executing intervention $d$ if intervention $d$ is executed are given by:

$$
S^{d}(t)=B^{d}(t)-R^{d}(t)-C^{d}(t)
$$

If $t=z, C^{d}(t)=C^{d}(z)$, otherwise, it equals to 0 .

The total net benefit between the $D S$ in which there is the possibility at $z$ to decide whether or not to execute intervention $d$ and the $D S$ in which there is the possibility at $z$ to decide whether or not to execute intervention $d^{*}$, (denoted as $\Delta$ ) at time 0 (analogous to the payoff in European call option) is given by:

$$
\Delta[z, S(z, T)]=e^{-\rho(T-z)} \mathbb{E} g\left(Z_{T}^{z, S^{d}(t)}\right)
$$

where, $\mathbb{E} g\left(Z_{T}^{z, S^{d}(t)}\right)$ is the expected value of:

$$
g(x)=\operatorname{Max}\left[S^{d}(z, T)-S^{d^{*}}(z, T), 0\right]
$$

The solution for Equation (8) has been extensively described in numerous references on applying Black and Scholes formulation. The explicit formulas to estimate the total net benefit are defined in following equations:

$$
\begin{aligned}
\Delta[z, & S(z, T)] \\
& =S(z, T) \Phi\left(d_{1}\right)-e^{-\rho(T-z)} S^{d^{*}}(z, T) \Phi\left(d_{2}\right)
\end{aligned}
$$


with

$$
\begin{gathered}
d_{1}=d_{2}+\sigma \sqrt{T-z} \\
d_{2}=\frac{\ln \left(\frac{S^{d}(z, T)}{S^{d^{*}}(z, T)}\right)+\left(\rho-\frac{1}{2} \sigma^{2}\right)(T-z)}{\sigma \sqrt{T-z}}
\end{gathered}
$$

where $\Phi(x)$ is the cumulative distribution function for normal standard distribution.

$$
\Phi(x)=\frac{1}{\sqrt{2 \pi}} \int_{-\infty}^{x} e^{\frac{-s^{2}}{2}} d s
$$

In order to find the optimal $z$, i.e. the OTD, it is necessary to solve the set of Equations (8) to (13). These equations, which involve the integral of an embedded stochastic process, i.e. the geometric Brownian motion, can be solved using the analytical and numerical approach suggested by Black and Scholes [10].

Although the formulation given above is the one to compute the expected total benefit of having the option to decide at time $z$ to execute an intervention or not, which is analogous to the determination of the payoff value in a European call option, it is possible to run simulations with different values of $z$ to compute how the value of the option to decide changes over time.

The use of this RO model to determine the OTD is demonstrated with a fictive example in the next section.

\section{Example}

\subsection{General}

In order to demonstrate the RO methodology a fictive example is done, in which it is imagined that the railway management organizations of a European freight corridor, e.g. Prorail in the Netherlands, DB in Germany, SBB and BLS in Switzerland, and RFI in Italy are the railway management organizations of the Rhine-Alp Corridor, need to determine when they will decide that interventions should be executed on the corridor or not. Each operator should make the decision at a time when it could be the most beneficial for them. The OTD for one organization over a 30-year time period is determined for a fictive railway link in the freight corridor. The link is used to transport 200,000 tons of goods per year $(\mathrm{N})$, and the annual growth is expected to be $0.5 \%$. The salvage value of the network is assumed to be 0 . 


\subsection{Global condition states}

The GCSs are given in Table 1. The condition state of each object in the link (e.g. track, bridges, tunnels, and the elements of which the objects are comprised such as ballast, sleepers, and rails) are discretely described in a range of 1 to 5, which is convenient for inspection and often used in practices.

Table 1: Global condition states (GCSs).

\begin{tabular}{|c|c|c|c|c|c|c|c|c|}
\hline \multicolumn{3}{|c|}{ GCS } & \multicolumn{3}{|c|}{0} & \multicolumn{3}{|c|}{1} \\
\hline \multicolumn{3}{|c|}{ Description } & \multicolumn{3}{|c|}{$\begin{array}{l}\text { All elements of all objects are in a } \\
\text { new or as-new condition state } \\
\text { (CS1). } \\
\text { The probability of the network } \\
\text { providing an adequate level of } \\
\text { service in the upcoming year is } \\
100 \% \text {. }\end{array}$} & \multicolumn{3}{|c|}{$\begin{array}{l}\text { All elements of all objects are in a } \\
\text { good condition state (CS2) or better. } \\
\text { The probability of the network } \\
\text { providing an adequate level of } \\
\text { service in the upcoming year is at } \\
\text { least } 90 \% \text {. }\end{array}$} \\
\hline \multirow{7}{*}{ 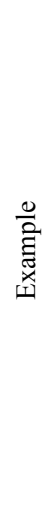 } & \multirow[t]{2}{*}{ 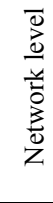 } & $\begin{array}{l}\vec{\nabla} \\
\text { స్తు }\end{array}$ & 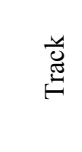 & 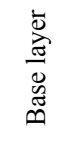 & 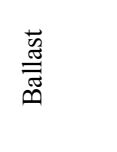 & 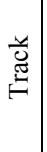 & 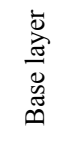 & 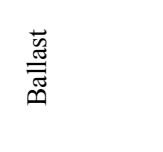 \\
\hline & & $\mathrm{CS}$ & 1 & 1 & 1 & 1 & 2 & 2 \\
\hline & \multirow{5}{*}{ 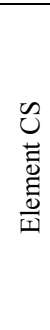 } & 1 & $\begin{array}{c}100 \\
\%\end{array}$ & $\begin{array}{c}100 \\
\%\end{array}$ & $\begin{array}{c}100 \\
\%\end{array}$ & $\begin{array}{l}1 \\
0 \\
0 \\
\% \\
\end{array}$ & $\begin{array}{c}90-99 \\
\%\end{array}$ & $\begin{array}{c}90-99 \\
\%\end{array}$ \\
\hline & & 2 & & & & & $\begin{array}{c}1-10 \\
\%\end{array}$ & $\begin{array}{c}1-10 \\
\%\end{array}$ \\
\hline & & 3 & & & & & & \\
\hline & & 4 & & & & & & \\
\hline & & 5 & & & & & & \\
\hline
\end{tabular}

\subsection{Decision strategies}

The possible DSs, i.e. the GCS that triggers a decision and the options for the organization at that time, are given in Table 2. For example, DS 1 refers to the strategy when the organization is to make a decision once the GCS1 is reached and the organization can decide to either execute an intervention or to do nothing.

Table 2: Decision strategies.

\begin{tabular}{|c|c|l|l|l|}
\hline \multirow{2}{*}{ DS } & Triggering & \multicolumn{3}{|c|}{ Intervention to be executed rather than do nothing intervention } \\
\cline { 3 - 5 } & GCSs & No. & \multicolumn{2}{|c|}{ Description } \\
1 & 1 & I-2 & $\begin{array}{l}\text { Minor intervention } \\
\text { level 1 }\end{array}$ & $\begin{array}{l}\text { Improve the base layer and geometric } \\
\text { condition of some but not all of the track }\end{array}$ \\
\hline 2 & 1 & I-3 & $\begin{array}{l}\text { Major intervention } \\
\text { level 1 }\end{array}$ & $\begin{array}{l}\text { Improve the base layers and geometric } \\
\text { condition of all of the track }\end{array}$ \\
\hline
\end{tabular}




\subsection{Deterioration and improvement}

The values of the parameters of the deterioration model at the start of the investigated time period and following the execution of each intervention are given in Table 3. For example, if the GCS1 is reached and intervention 2 is executed then the deterioration model to be used following execution of the intervention will have the parameter values $\alpha=0.002$ and $\mathrm{m}=2.200$. The effectiveness of the interventions are represented through the values of the deterioration model parameters (the parameter values for the do-nothing intervention are given as $\mathrm{t}=0$ values corresponding to each GCS and can also be seen in Table 3).

Table 3: Values of deterioration model parameters following the execution of interventions.

\begin{tabular}{|c|c|c|c|}
\hline Parameters & \multicolumn{3}{|c|}{ GCS1 } \\
\cline { 2 - 4 } & $\mathrm{t}=0$ & $\mathrm{I}-1$ & $\mathrm{I}-2$ \\
\hline$\alpha$ & 0.002 & 0.002 & 0.002 \\
\hline$m$ & 2.500 & 2.200 & 2.100 \\
\hline
\end{tabular}

It is important to note that the restoration of infrastructure to an as-new condition does not necessarily mean that the deterioration of the infrastructure following the execution of the intervention deteriorates is the same as the newly built infrastructure. The three cases are:

- The deterioration may be the same, e.g. all objects are replaced with objects that are identical to the original objects.

- The deterioration may be faster, e.g. some objects are replaced with objects identical to the original objects but others are not replaced and, therefore, have a higher failure rate than they did at $\mathrm{t}=0$.

- The deterioration may be slower, e.g. all objects are replaced with objects that deteriorate more slowly than the original objects, which is something that may happen due to the consideration of information gathered over the time period since the construction of the original objects in the design of the new objects and the integration of new technologies.

The interventions assumed in this example correspond to the third case.

The changes in the probabilities of not reaching GCS are shown in Figure 2. These changes are shown assuming no interventions are executed (the lower curve), and for one possible future scenario, assuming each DS was followed (the two upper curves) and either intervention 1 or intervention 2 was executed. In Figure 2 it can be seen, for example, that if a decision is to be made in year 13 as to which intervention is to be executed there is a $30 \%$ chance that the link will not yet have reached GCS1. At that point in time the organization will be able to decide to execute the do-nothing intervention, or to execute a minor intervention or major intervention, i.e. to follow DS0, DS1 or DS2. 


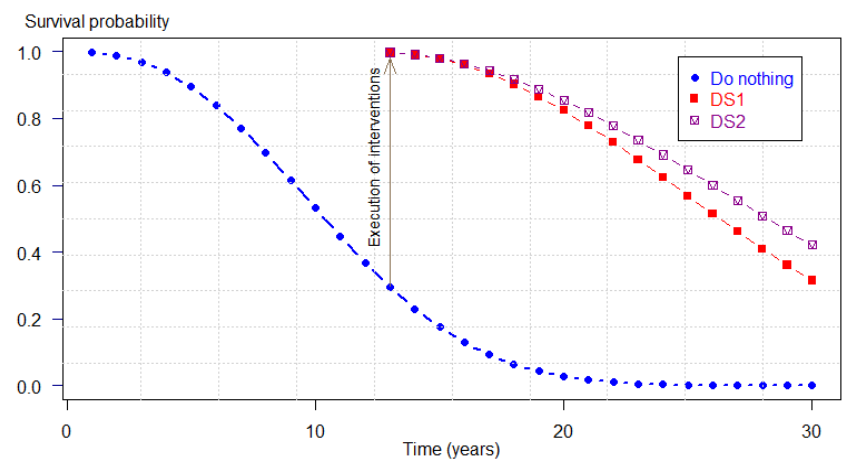

Figure 1: $\quad$ Probabilities of being in each GCS if each IS is followed.

\subsection{Impacts}

It is assumed that impacts are incurred in three general time periods from the start of the investigated time period to the execution of the first planned intervention, during the execution of planned interventions, and after the execution of planned interventions.

The impacts that are incurred are grouped as follows:

- during the execution of unplanned interventions, $W$, which have an increasing probability of happening as the time between interventions increases, and

- during the execution of planned interventions, $C$,

- between interventions due to routine maintenance, $R$,

- between interventions due to normal operation, $O$.

The parameters of the models of the impacts are given in Table 4. For example, if an intervention is executed when the link enters GCS1, it is assumed that an impact of $0.4 \times 106 \mathrm{mu}$ due to schedule restrictions (W), an impact of $1.6 \times 106 \mathrm{mu}$ due to the execution of the intervention $(\mathrm{C})$, an impact of $0.22 \times 106 \mathrm{mu} /$ year on average due to routine maintenance $(\mathrm{R})$ until the next intervention and an impact of $0.72 \times 106 \mathrm{mu} /$ year due to normal operation $(\mathrm{O})$ since the last intervention. Following the execution of an intervention, $\mathrm{R}$ and $\mathrm{O}$ are assumed to increase deterministically annually by $1 \%$ and $1.5 \%$, respectively.

Table 4: Impact model parameters.

\begin{tabular}{|c|c|c|c|c|}
\hline \multirow{2}{*}{ Parameters } & \multirow{2}{*}{ Unit } & \multicolumn{3}{|c|}{ GCS1 } \\
\cline { 3 - 5 } & & $t=0$ & $I-2$ & $I-3$ \\
\hline$W$ & $10^{6} \mathrm{mu}$ & 0.400 & 0.400 & 0.400 \\
\hline$C$ & $10^{6} \mathrm{mu}$ & 0 & 1.600 & 1.820 \\
\hline$R$ & $10^{6} \mathrm{mu} /$ year & 0.200 & 0.220 & 0.213 \\
\hline$O$ & $10^{6} \mathrm{mu} /$ year & 0.720 & 0.720 & 0.720 \\
\hline
\end{tabular}


For further example, in year 13 a decision will be made to do nothing or to execute intervention 1 or 2 , depending on the condition of the infrastructure in year 13 and the expected condition state of the infrastructure from year 13 to $\mathrm{T}$. If either intervention 1 or 2 is executed the link will be restored to an as-new condition and impacts $C$ will be incurred, where $C$ is larger for intervention 2 as it is more extensive $\left(1.6 \times 10^{6} \mathrm{mu}\right.$ vs. $\left.1.82 \times 10^{6} \mathrm{mu}\right)$. In both cases $O$ and $W$, which are the same as they depend on the time to arrival in the triggering GCS and are independent of the type of intervention executed, will be incurred. In both cases $\mathrm{R}$ will be incurred but the value of $R$ will be higher if intervention 1 is executed than if intervention 2 is executed. This is because if a minor intervention is executed the routine maintenance costs will be higher following the intervention than if a major intervention is executed.

The value of each impact type $h(t)$ in Equation (5) is modeled as a geometric Brownian motion (Equation (2)), with a drift parameter $\mu=0.0003$ and standard deviation $\sigma=0.2$. A discount rate of $\rho=2 \%$ is used. The input was then used with the model described in section 3 to obtain the results in section 4.2.

\subsection{Results}

The expected benefit of waiting to determine if an intervention should be executed if each of the DSs are followed, i.e. the option value (Equation (8)), is given in Figure 2 and Table 5. The option value is the difference between the benefits if the DS is followed and the benefits if the do-nothing reference strategy is followed. Each point in each curve represents the option value of deciding to execute or not execute an intervention at time $z$ in the investigated period of 30 years. For example, if DS2 is followed and the decision is made in year 7 whether to execute the intervention or not, the expected benefit will be $8.9 \times 10^{6} \mathrm{mu}$ (point $\mathrm{C}$ in Figure 2). If DS2 is followed and the decision is made in year 15 whether to execute the intervention or not, the expected benefit is $14.73 \times 10^{6} \mathrm{mu}$ (point A in Figure 2). If DS2 is followed and the decision is made in year 20 whether to execute the intervention or not, the expected benefit is $11.24 \times 10^{6} \mathrm{mu}$ (Point B in Figure 2).

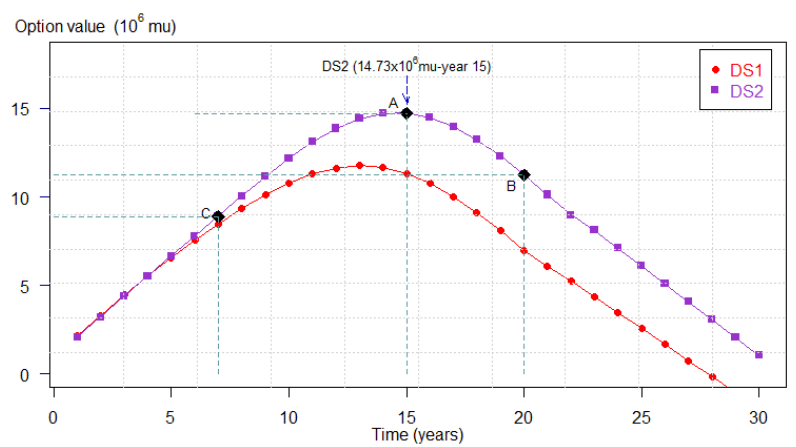

Figure 2: Option values of the investigated decision strategies. 
Table 5: Optimal times to decide and option values of decision strategies.

\begin{tabular}{|c|c|c|c|}
\hline Strategies & Ref & 1 & 2 \\
\hline $\begin{array}{c}\text { Maximum expected benefits } \\
\left(10^{6} \mathrm{mu}\right)\end{array}$ & 769.84 & 781.60 & 784.56 \\
\hline $\begin{array}{c}\text { Optimal time to decide } \\
(\text { years })\end{array}$ & $\mathrm{NA}$ & 13 & 13 \\
\hline $\begin{array}{c}\text { Maximum option value per strategy } \\
\left(10^{6} \mathrm{mu}\right)\end{array}$ & $\mathrm{NA}$ & 11.76 & 14.72 \\
\hline $\begin{array}{c}\text { Expected benefits if decision is } \\
\text { to be made in year 15 } \\
\left(10^{6} \mathrm{mu}\right)\end{array}$ & $\mathrm{NA}$ & 781.17 & 784.56 \\
\hline $\begin{array}{c}\text { Option value if decision is to be made in year } 15 \\
\left(10^{6} \mathrm{mu}\right)\end{array}$ & $\mathrm{NA}$ & 11.33 & 14.72 \\
\hline
\end{tabular}

In this example, both DSs yield more benefit than the do-nothing reference strategy. The OTD depends on the type of intervention to be executed and the probability of the link being in the GCS in which it would be decided to execute an intervention. In this example, the highest option value is obtained from DS2 when the decision to execute an intervention is made in year 15.

The general increase in option values that occurs following time 0 is due to the increased information that the railway management organization receives and, therefore, upon which the decision to intervene can be based. The main reasons for the decrease in option values that occurs towards the end of the 30 -year period are due to the decreased number of years in which benefits can be obtained if an intervention is executed and the increased speed of deterioration, which greatly increases the costs of unplanned interventions (W). Thus, the expected benefit at $\mathrm{t}=30$ is 0 .

It is noted that, in general, DS2 yields higher option values than DS1. For example, if the decision is to be made in year 15, DS2 yields a higher option value $\left(14.72 \times 10^{6} \mathrm{mu}\right)$ than DS1 $\left(11.33 \times 10^{6} \mathrm{mu}\right)$. The expected benefit at $\mathrm{t} \geq 27$ of DS1 is 0 , which infers that no benefit can be obtained from executing an intervention after year 27.

\section{Conclusions}

In this paper, a real option methodology is presented for the determination of the optimal expected time in the future for a railway infrastructure manager to decide what types of interventions, if any, are to be executed. Such a methodology is particularly useful in the management of railway infrastructure that belongs to a multinational freight corridor, where multiple railway management organizations are involved, and every time one executes an intervention it can affect the flow of trains on the part of the infrastructure controlled by others. The real option methodology can be used in addition to the conventional life cycle cost estimation techniques that have been so far used in practice. This will help managers to have better ideas and options so he/she can make good management decisions. The methodology is demonstrated by determining the optimal time to 
decide to execute interventions on a fictive rail corridor for a railway management organization. The additional benefits that managers can gain is the option values compared to the do-nothing strategies or compared to other strategies that managers can decide to execute.

\section{References}

[1] O. Ivanova, B. V. Zeebroeck and K. Karel Spitaels, "Optimal infrastructure charging in a multi country railway corridor," in European Transport Conference, 2008.

[2] S. Santa-Cruz and E. Heredia-Zavoni, "Maintenance and decommissioning real options models for life-cycle cost-benefit analysis of offshore platforms," Struct. Infrastruct. Eng., vol. 7, no. 10, pp. 733-745, Oct. 2011.

[3] H. T. J. Smit, "Infrastructure Investment as a Real Options Game: The Case of European Airport Expansion," Financ. Manag., vol. 32, no. 4, p. 27, 2003.

[4] P. M. Pimentel, J. Azevedo-Pereira, and G. Couto, "High-speed rail transport valuation," Eur. J. Financ., vol. 18, no. 2, pp. 167-183, 2012.

[5] B. Agusdinata, "Exploratory analysis to support real options analysis: an example from electricity infrastructure investment," in Systems, Man and Cybernetics, 2005 IEEE International Conference on, 2005, vol. 4, pp. 3689-3696 Vol. 4.

[6] D. N. Ford, D. M. Lander, and J. J. Voyer, "A Real Options Approach to Valuing Strategic Flexibility in Uncertain Construction Projects," Constr. Manag. Econ., vol. 20, pp. 343-351, 2002.

[7] B. W. Schlake, C. P. L. Barkan, and J. R. Edwards, "Train Delay and Economic Impact of In-Service Failures of Railroad Rolling Stock," Transp. Res. Rec. J. Transp. Res. Board, no. 2261, 2011.

[8] K. Kobayashi, K. Kaito, and N. Lethanh, "Deterioration Forecasting Model with Multistage Weibull Hazard Functions," J. Infrastruct. Syst., vol. 16, no. 4, pp. 282-291, 2010.

[9] B. Dodson, The Weibull Analysis Handbook. Quality Press, American Society for Quality, 2006.

[10] F. Black and M. Scholes, "The Pricing of Options and Corporate Liabilities," J. Polit. Econ., vol. 81, no. 3, pp. 637-654, 1973.

[11] M. G. H. Bell and C. Cassir, Reliability of Transport Networks. Baldock, Hertfordshire, England: Research Studies Press, 2001. 